\title{
ESTUDOS DE MÉTODOS PARA AVALIAÇÃO CEGA DE QUALIDADE DE IMAGENS
}

\author{
Wagner Ferreira de Barros ${ }^{1}$ \\ Flávio Luis Cardeal Pádua ${ }^{2}$ \\ Gustavo Augusto Silva Junqueira ${ }^{3}$ \\ Rafael Sales Medina Ferreira ${ }^{4}$
}

\begin{abstract}
RESUMO
Avaliação da qualidade da imagem é a medição de um atributo (propriedades ou características) de uma determinada imagem. Esta é utilizada em diversas aplicações na área de processamento de imagens. A qualidade da imagem pode ser avaliada por duas metodologias distintas, forma subjetiva ou objetiva. O método objetivo quantifica a qualidade da imagem de forma automática, havendo ou não a imagem ideal de referência. $O$ método subjetivo faz uso da opinião de um observador, sendo este um método mais caro. Este artigo apresenta uma revisão de métodos objetivos sem referência da avaliação da qualidade de imagem. Foram abordadas as principais características dos algoritmos de evolução, neural e de Ruídos e Borramento, todos sem a utilização de uma imagem como referência. Baseandose nos resultados satisfatórios apresentados, o estudo e comparação destas métricas podem ser aprofundados de forma prática em futuros trabalhos.
\end{abstract}

Palavras-chave: Avaliação da Qualidade de Imagem (AQI). Método objetivo. Método sem referência. Algoritmo evolutivo. Algoritmo neural. Borramento e ruído.

\section{STUDIES OF METHODS FOR BLIND IMAGE QUALITY ASSESSMENT}

\begin{abstract}
Image Quality Evaluation is the measurement of an attribute (properties or characteristics) of a given image. It is used in several applications in the field of image processing. The quality of the image can be evaluated in two different methods: subjective and objective. The objective method quantifies the image quality automatically, with or without the ideal image reference. The subjective method uses the opinion of an observer; however, it is more expensive. This paper presents a review of the objective method with no reference of the image quality evaluation. The survey covered the main features of evolutionary, neural and Noise and Blurring algorithms. All of them had no use of an image as reference. Based on the satisfactory results presented, the study and the comparison of these metrics may undergo a more practical approach in future works.

\footnotetext{
${ }^{1}$ Doutorado em Ciências da Computação pela Universidade Federal de Minas Gerais. Coordenador e professor do Curso Técnico em Informática CEFET-MG Campus Varginha. E-mail: wbarros@gmail.com

${ }^{2}$ Doutorado em Ciências da Computação pela Universidade Federal de Minas Gerais. Professor de Engenharia de Computação do CEFET-MG Campus Belo Horizonte. E-mail: cardeal@decom.cefetmg.br

${ }^{3}$ Graduando em Engenharia de Computação pelo CEFET-MG. E-mail: gustavoasj@gmail.com

${ }^{4}$ Graduando em Engenharia de Computação pelo CEFET-MG. E-mail: rafasale93@gmail.com
} 
Keywords: Image Quality Evaluation (IQA). Objective method. No reference method. Evolutionary algorithm. Neural algorithm. Blur and noise.

\section{INTRODUÇÃO}

A qualidade de uma imagem é talvez o fator mais importante em suas mais diversas aplicações e finalidades. Ela pode ser afetada pela perda de características resultantes de processos como: compactação, compartilhamento e armazenamento.

A avaliação da qualidade de uma imagem se divide, primeiramente, em duas classes maiores, quais sejam: a avaliação subjetiva e a avaliação objetiva. No método subjetivo, a avaliação da qualidade é realizada, propriamente, por um ou mais observadores. O ser humano é especialista na avaliação da qualidade de imagens, conseguindo, por exemplo, perceber pequenas variações de contraste e foco, e assim, avaliar se uma imagem é boa ou ruim. Tal abordagem apresenta resultados confiáveis, porém sua aplicação prática é limitada. Por exemplo, uma das principais aplicações envolvendo o uso de métricas de qualidade de imagem está diretamente relacionada a sistemas automáticos de restauração de imagens. Em tais situações, faz-se necessário obter algum dado objetivo que indique a qualidade da imagem (GONZALEZ; WOODS, 2000).

Métodos objetivos medem a qualidade da imagem automaticamente, produzindo um dado numérico que quantifica a qualidade de uma ou mais características específicas da imagem. Uma métrica objetiva pode ser considerada satisfatória quando produz os mesmos resultados que um método subjetivo, dadas as mesmas circunstâncias relacionadas à imagem.

O principal objetivo deste artigo é mostrar alguns métodos objetivos sem referência de avaliação de imagens já existentes, trazendo uma breve explicação do funcionamento das mesmas e os resultados obtidos em cada uma. Foi construída, também, uma taxonomia mostrando como podem ser subdivididos os métodos de avaliação, com foco nas métricas sem referência.

\section{MÉTRICAS OBJETIVAS}

As métricas objetivas, com base na disponibilidade da imagem ideal, podem ser classificadas em full-reference (FR), reduced-reference (RF) e no-reference (NR). Neste trabalho, enfatizamos as métricas sem referência (NR), sendo que a qualidade das imagens é 
mensurada entre um intervalo fechado de valores [0..1], onde 0 é ruim e 1 é ótimo (THUNG; RAVEENDRAN, 2009).

A abordagem conhecida como full-reference, avalia a qualidade de certa imagem utilizando outra imagem como referência, leia-se, a imagem original. Este método é o mais comum, porém nem sempre é o ideal. O principal problema das métricas de full-reference é que em muitos casos não há uma imagem original a ser utilizada como referência. Uma das métricas mais conhecidas da avaliação com imagem de referência é a Peak Signal-to-Noise Ratio (PSNR) (DIMAURO, 2012). Este tipo de avaliação quantifica a melhoria da imagem restaurada em relação à imagem original, sendo que os valores positivos indicam uma melhoria na imagem, os valores negativos indicam uma deterioração e os valores nulos indicam que não houve modificação na imagem.

A abordagem com referência reduzida ou reduced-reference, avalia a imagem deteriorada e compara os resultados com algumas características da imagem original.

Os métodos que não utilizam nenhuma referência, conhecidos como "no-reference" ou "blind assessment", utilizam algoritmos que retornam índices de fator de qualidade. Estes índices correspondem a características mensuráveis da imagem (contraste, integridade das bordas, alcance de borramento e nível de ruído), ao contrário das que retornam resultados comparativos onde se tem uma imagem de referência. Comparado aos outros dois métodos, este último é o menos abordado, por ser mais complexo. As métricas que não utilizam referência produzem os resultados esperados, mas esses resultados devem ser analisados, como, por exemplo, medindo a presença de ruído na imagem. A presença de ruído em uma imagem nem sempre indica que a mesma está ruim. As métricas sem referência medem apenas a quantidade do ruído, necessitando de um meio para dizer se essa quantidade é prejudicial à qualidade ou não.

As métricas sem referência se dividem em diversas pequenas áreas, sendo que cada uma delas se especializa em um método diferente para fazer a avaliação da qualidade da imagem.

A seguir, apresentamos a classificação proposta para as métricas objetivas abordadas neste trabalho. 


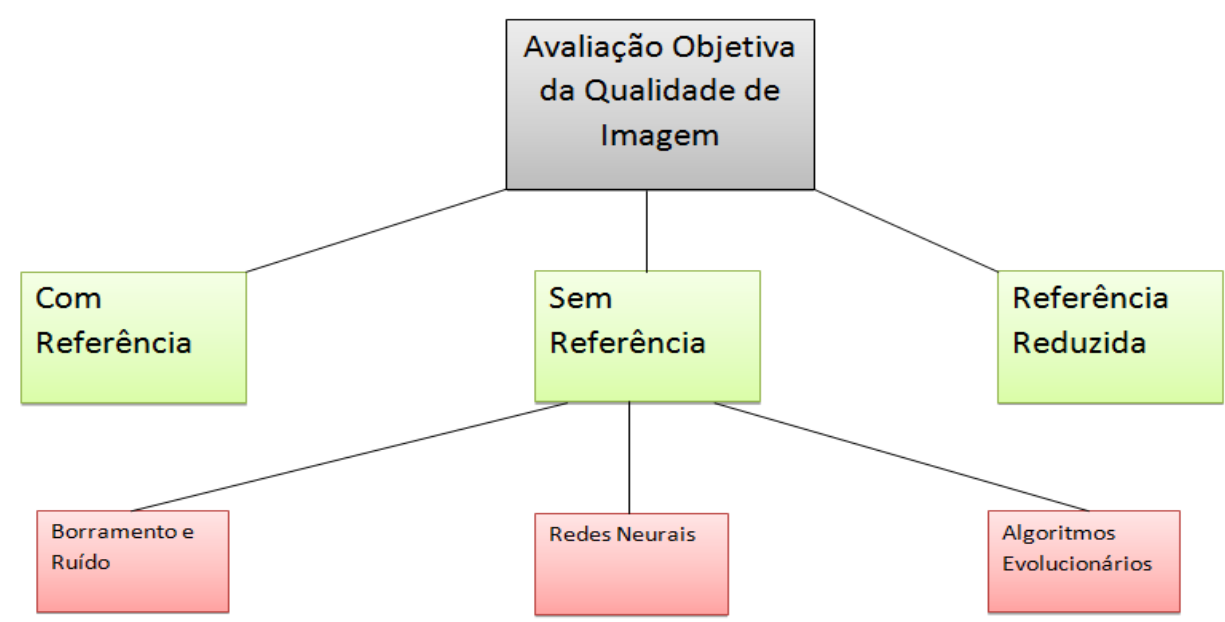

Figura 1 - Taxonomia da Avaliação objetiva da qualidade da imagem, baseada no resultado do estudo. Fonte: Autor

\subsection{Algoritmos evolucionários}

São algoritmos muito utilizados em métodos de busca e otimização, os quais são inspirados na evolução biológica. Os dados de entrada são chamados de "cromossomos" e a cada geração são escolhidos os melhores resultados para serem usados em uma geração posterior.

O método de máquina classificadora utiliza algoritmos evolucionários para obter uma boa aproximação da imagem ótima (SURESH, 2009).

O algoritmo evolucionário funciona da seguinte forma: é organizado em iterações (gerações). Na maioria dos casos, em cada geração, um conjunto de candidatos à solução (população) é usado para gerar um conjunto novo de soluções melhores, usando operações evolucionárias específicas como seleção, cruzamento e mutação. Este novo conjunto de candidatos à solução substitui (parcialmente ou totalmente) a geração anterior.

Primeiramente, são extraídas características da imagem, como mostrado na figura 2.

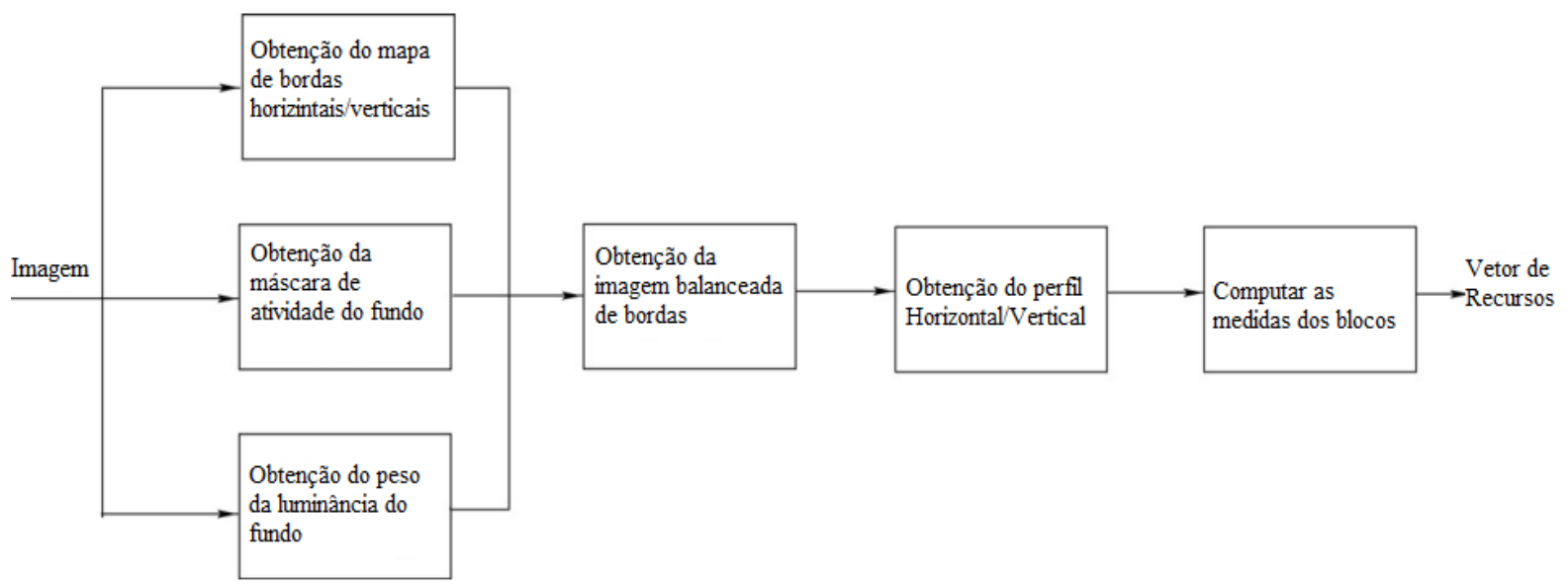

Figura 2 - Visão geral da fase de extração da característica da imagem Fonte: Adaptado de Suresh (2009) 
Os valores iniciais do algoritmo evolucionário são valores aleatórios. O objetivo deste algoritmo é relacionar estes valores aleatórios com as amostras extraídas para se obter um resultado o mais próximo do mean opinion score (MOS) (GEORGE, 2013), que é o resultado de métodos subjetivos. Já o algoritmo evolucionário é utilizado para encontrar esta relação.

O algoritmo utiliza operadores genéticos, os quais são operadores de seleção, avaliação, mutação e cruzamento.

Os passos utilizados no algoritmo evolucionário estão descritos abaixo:

1. Cria uma população aleatória.

2. Calcula a aptidão destes valores.

3. Seleciona os pais para os operadores genéticos.

4. Gera uma nova população utilizando os operadores genéticos.

5. Se os critérios de parada são satisfeitos, então para. Se não, volta ao passo 2.

Os parâmetros do algoritmo são: o tamanho da população (tp), a probabilidade de seleção ( $p s)$, a probabilidade de cruzamento $(p c)$ e a probabilidade de mutação $(p m)$. O critério de parada M é o numero máximo de gerações.

Os parâmetros utilizados no algoritmo evolucionário da máquina classificadora foram: $\mathrm{tp}=30, \mathrm{ps}=0.08, \mathrm{pc}=0.6, \mathrm{pm}=0.05$ e o número máximo de gerações é 500 . Estes valores foram selecionados pela tentativa e erro.

Em experimentos que utilizaram os parâmetros acima, obteve-se um resultado significativo, sendo que foram executados 50 testes com diferentes tipos de imagens. $\mathrm{O}$ algoritmo obteve um resultado de 0.923 onde 0 é ruim e 1 é ótimo. Comparando com o resultado de 0.927 da métrica com referência structural similarity image quality metric (SSIM), ambos são próximos de um, portanto emulando muito bem o MOS.

\subsection{Algoritmos Neurais}

Redes Neurais Artificiais são técnicas computacionais que apresentam um modelo matemático inspirado na estrutura neural de organismos inteligentes e que adquirem conhecimento através da experiência (CARDON; MULLER, 1994).

A propriedade mais importante das redes neurais é a habilidade de aprender em seu ambiente e com isso melhorar seu desempenho. Isso é feito através de um processo iterativo de ajustes aplicado a seus pesos, qual seja: o treinamento. 
O aprendizado ocorre quando a rede neural atinge uma solução generalizada para uma classe de problemas.

O método sem referencia (NR) de aprendizado sequencial para o cultivo e poda rede de função de base radial (GAP-RBF) (VENKATESH BABU, 2007), é utilizado para avaliar a qualidade das imagens codificadas por JPEG.

Este método, em sua primeira parte, leva em consideração fatores de sensibilidade do sistema visual humano, como: amplitude de borda, comprimento de borda, atividade e luminância do fundo. A partir da imagem de teste se obtém os valores destas características.

Uma vez que a qualidade da imagem é um fenômeno subjetivo, o observador humano desempenha um papel importante na qualidade da imagem testando a métrica. A prova subjetiva designa a opinião de um espectador (opinion score) em uma determinada imagem com base em como ela é percebida. O mean opinion score MOS é a pontuação média da opinião sobre todos os assuntos. O objetivo de qualquer métrica de qualidade é prever a qualidade tão perto quanto possível do MOS.

Portanto, o objetivo aqui é encontrar a relação funcional entre as características HVS extraídos e MOS para quantificar a qualidade de imagem. Para calcular a relação desconhecida, a rede de GAP-RBF é usada. Este algoritmo de aprendizagem sequencial é melhor do que os algoritmos de aprendizagem, pois não necessita de reciclagem sempre que novas observações são recebidas.

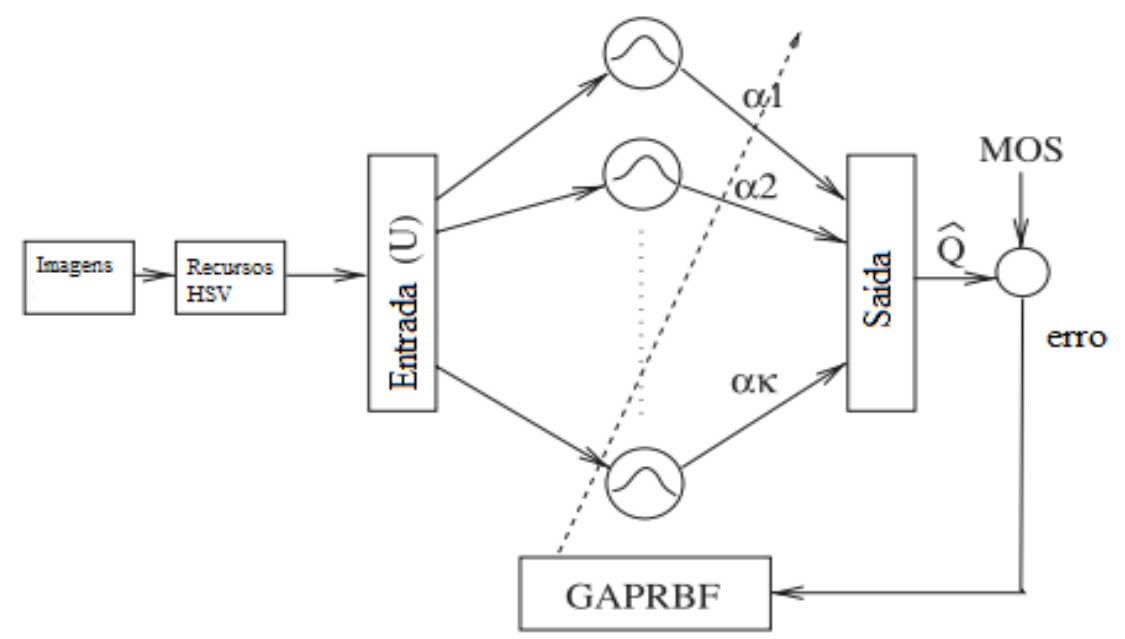

Figura 3 - Visão geral do algoritmo de estimativa de qualidade de imagem proposto Fonte: Adaptado de Venkatesh Babu (2007)

Onde $\alpha$ é o peso de cada conexão que interliga os neurônios de entrada e saída.

Neste algoritmo, os critérios para o cultivo / poda de neurônios escondidos são baseados na importância dos neurônios para a saída da rede. O algoritmo apenas atualiza os 
parâmetros do neurônio mais próximo para minimizar o erro de aproximação. Assim, este método é econômico computacionalmente e em requisitos de memória.

Após testes serem feitos com diversos tipos de imagens, o algoritmo produz um resultado de 0.9564 , sendo que 0 é ruim e 1 é ótimo. Por obter o valor mais próximo de um, este resultado é considerado satisfatório.

\subsection{Borramento e ruído}

Borramento e ruído são duas características básicas de uma imagem, cuja quantidade afeta diretamente sua qualidade. O borramento, em especial, pode ter várias causas, como a compressão de uma imagem, a falta de foco ou mesmo em um processo para redução do ruído. Por sua vez, o ruído pode ser causado durante a obtenção da imagem, dependendo do modo como a luz percorre na sua formação.

Choi, Jung e Cheon apresentam um método de avaliação cega, baseado na quantidade de borramento e ruído contidos em uma imagem. Neste método, se assume que o borramento e o ruído são os principais fatores do decaimento da qualidade de uma imagem. A figura 4 é um diagrama apresentando a métrica. A ideia do método é medir tanto o borramento quando o ruído separados, e a partir dessa mediação, analisar os resultados em conjunto.

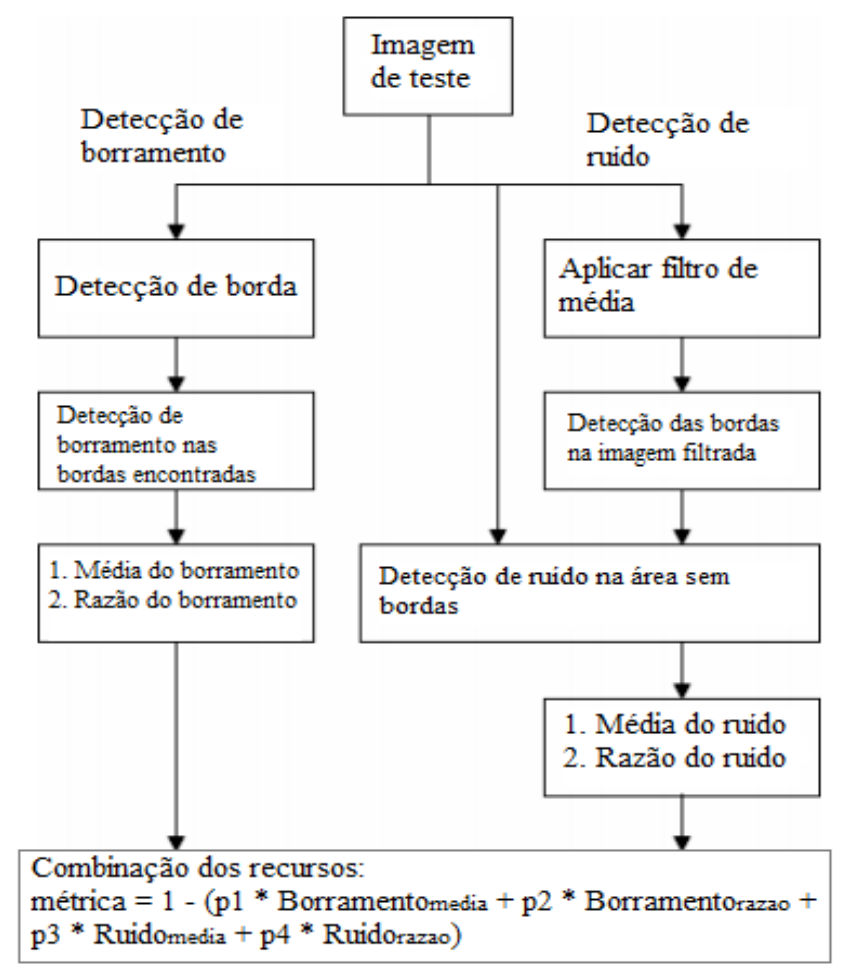

Figura 4 - Diagrama do modelo proposto Fonte: Adaptado de Choi, Jung e Jeon (2009). 
No caso do borramento, são duas etapas de análise. Primeiro, ocorre a detecção das bordas da imagem. Depois, a análise do borramento nessas bordas. Essa análise é feita a partir da diferença de intensidade entre o pixel atual e a média da intensidade de seus pixels vizinhos. Será considerado que há borramento na imagem se essa diferença for muito baixa (CHOI; JUNG, CHEON, 2009).

Já o ruído, também precisa da detecção das bordas, porém primeiro é aplicado um filtro para retirar todo o ruído da imagem e então detectar as bordas. Esse processo se faz necessário porque o ruído pode afetar a detecção de bordas. Após a detecção, o ruído será encontrado nas áreas em que não são de bordas na imagem que está sendo testada.

Cada um desses processos resulta em dois valores: a média e a razão do borramento e do ruído. A métrica, enfim, propõe uma equação linear, que tem um gasto computacional significativamente baixo.

\footnotetext{
métrica $=1-\left(\mathbf{P 1} *\right.$ Borramento $_{\text {médio }}+\mathbf{P 2} *$ Borramento $_{\text {razão }}+\mathbf{P 3} *$ Ruído $_{\text {médio }}+\mathbf{P 4}$ * Ruído orazão)
}

$P 1, P 2, P 3$ e $P 4$ são os pesos de cada dado. Após exaustivos testes, os parâmetros foram otimizados para $\boldsymbol{P 1}=1, \boldsymbol{P 2}=0.95, \boldsymbol{P 3}=0.3$ e $\boldsymbol{P 4}=0.75$. Esses pesos mostram que o borramento tem um efeito maior na percepção da qualidade da imagem.

Os resultados encontrados por Choi, Jung e Jeon foram considerados satisfatórios pelos autores, tendo a métrica se destacado como um meio satisfatório de avaliação cega, quando comparada ao método PSNR.

\subsubsection{Nível de detalhe}

Um outro método que parte dessas características da imagem e foi bastante endossado por seus autores, Dosselmann e Yang, como sendo muito eficiente, é a avaliação de imagens por seu nível de detalhe. Nível de detalhe é a medida da variação dos pixels em uma imagem. Se esse valor varia bastante, a imagem tem uma alta quantidade de detalhes. O contrário é observado quando o valor tem variações baixas. Esse método leva em consideração a compressão da imagem, além do borramento e ruído (DOSSELMANN; YANG, 2011).

Como a variação não é constante sobre a imagem, avalia-se a imagem por partes. A divisão da imagem se dá por várias regiões ou blocos de forma retangular. São apresentados 
diferentes métodos para medir o nível de detalhe em cada um desses blocos da imagem e também métodos que avaliam cada uma das características citadas pelos autores. Assim, é possível obter a quantidade de borramento, ruído e compressão dos blocos e seu efeito no nível de detalhe.

\section{CONCLUSÃO}

Apesar de existirem diferentes formas de uma imagem perder qualidade, existem também, diferentes formas de avaliar a mesma. De maneira geral, estes métodos de avaliação baseiam-se no método mais básico, que é a avaliação subjetiva. A avaliação também se dá, normalmente, através da comparação da imagem cuja qualidade se quer determinar com outra, com a qualidade já avaliada.

Neste artigo, porém, foram coletadas e apresentadas outras métricas, cujo estudo não é tão aprofundado quanto ao das anteriormente mencionadas. Essas são métricas objetivas, mas que não utilizam uma imagem para comparação. Cada uma foi apresentada com uma breve explicação de como funciona. Ao fim, pode-se perceber que a avaliação objetiva sem referência é uma área ainda em expansão, contendo, ainda, diferentes áreas menores, que representam cada método de avaliação.

Todas as métricas apresentadas neste artigo tiveram um resultado satisfatório. $\mathrm{O}$ objetivo de conhecer e explicitar métricas de avaliação cega foi atingido. O foco, a partir de então, será na aplicação e comparação dos resultados destas e possivelmente de outras métricas, dadas as mesmas circunstâncias.

\section{REFERÊNCIAS}

CARDON, A.; MULLER, D. N. Introdução às redes neurais artificiais. 1994. Dissertação (Curso de Pós Graduação e Ciência da Computação) Instituto de Informática, Universidade Federal do Rio Grande do Sul: Porto Alegre, 1994.

CHOI, M. G.; JUNG, J. H.; JEON, J. W. No-Reference image quality assessment using Blur and Noise. World Academy of Science, Engineering and Technology, n. 12, v. 28. p 163167, jan. 2009.

DIMAURO,G. A new image quality metric based on human visual system. In: IEEE INTERNATIONAL CONFERENCE ON VIRTUAL ENVIRONMENTS HUMANCOMPUTER INTERFACES AND MEASUREMENT SYSTEMS (VECIMS), 2012, Tianjin. Proceedings... Disponível em: < http://ieeexplore.ieee.org/xpl/articleDetails.jsp? arnumber=6273216>. Acesso em: 16 jun. 2013. 
DOSSELMANN, R.; YANG, X. D. No-Reference image quality assessment using Level-ofDetail, 2011. Department of Computer Science, University of Regina, Regina, Canadá, maio 2011.

GEORGE, A. G.; PRABATHY, A. K. A Survey On different approaches used in image quality assessment. International Journal of Emerging Technology and Advanced Engineering, Coimbatore, India, n. 2, v. 3, fev. 2013.

GONZALEZ, R. C.; WOODS, R. Processamento de imagens digitais. 1. ed. São Paulo: Edgard Blucher, 2000.

SURESH, S. No-reference image quality assessment using modified extreme learning machine classifier. Applied Soft Computing, Seoul, n. 2, v. 9. p. 541-552, mar. 2009.

THUNG, K. H.; RAVEENDRAN, P. A survey of image quality measures. In:

INTERNATIONAL CONFERENCE FOR TECHNICAL POSTGRADUATES (TECHPOS), 2009, Kuala Lumpur. Proceedings... Disponível em: < ieeexplore.ieee.org/xpl/ articleDetails.jsp?arnumber=5412098>. Acesso em: 21 mar. 2013.

VENKATESH BABU, R. No-reference JPEG-image quality assessment using GAP-RBF. Signal Processing, Singapura, p. 1493-1503, jun. 2007.

Recebido em: 15/09/2013

Aprovado em: 05/11/2013 